\title{
Chronic Physical Activity Does Not Impact Metabolic Responses to Low or High Doses of Resistant Starch: A Crossover Trial
}

\author{
Jiyoung S. Kim 1,2, Sam R. Emerson',3, Mark D. Haub1, Sara K. Rosenkranz ${ }^{1}{ }^{*}$ \\ ${ }^{1}$ Department of Food, Nutrition, Dietetics, and Health, College of Health and Human Sciences, Kansas State University, \\ Manhattan, KS, USA \\ ${ }^{2}$ Department of Food and Nutrition, University of Georgia, Athens, GA, USA \\ ${ }^{3}$ Department of Nutritional Sciences, Oklahoma State University, Stillwater, OK, USA \\ Email: *SaraRose@ksu.edu
}

How to cite this paper: Kim, J.S., Emerson, S.R., Haub, M.D. and Rosenkranz, S.K. (2019) Chronic Physical Activity Does Not Impact Metabolic Responses to Low or High Doses of Resistant Starch: A Crossover Trial. Open Journal of Preventive Medicine, 9, 69-79.

https://doi.org/10.4236/ojpm.2019.97007

Received: June 26, 2019

Accepted: July 22, 2019

Published: July 25, 2019

Copyright (C) 2019 by author(s) and Scientific Research Publishing Inc. This work is licensed under the Creative Commons Attribution International License (CC BY 4.0).

http://creativecommons.org/licenses/by/4.0/

\begin{abstract}
Physical activity and resistant starch are known independently, to beneficially affect metabolic health especially in the gut. However, little is known about the combined effects of physical activity and resistant starch. Thus, the purpose of this study was to investigate the effects of physical activity at different dosages of resistant starch on gut and metabolic health, represented by breath hydrogen and blood glucose responses. Twenty young, healthy participants were stratified into two physical activity groups based on seven-day accelerometry data. Participants visited the lab twice in random order and consumed a meal with either $5 \mathrm{~g}$ or $25 \mathrm{~g}$ of resistant starch. Breath hydrogen and blood glucose were measured at baseline and serially for six and two hours after meal consumption respectively. Total area under the curve (AUC) for breath hydrogen and incremental AUC for blood glucose were not different between physical activity groups or resistant starch conditions. Thus, chronic physical activity status did not impact breath hydrogen or blood glucose responses to either low or high resistant starch meals.
\end{abstract}

\section{Keywords}

Dietary Fiber, Physical Activity, Resistant Starch, Fermentation, Diabetes

\section{Introduction}

The association between gut microbiota and chronic diseases is becoming well established [1]. Microbiota primarily live in the large intestine, and dysbiotic microbiota has been found to be associated with diabetes, heart disease, and ob- 
esity [2]. A gut microbial population with higher diversity and a lower ratio of Firmicutes to Bacteridetes has been found to be an indicator of a healthy state of the gut [3] [4]. For analyzing gut status, a direct measure of microbiota analysis via cecum/feces has been widely applied to examine the association between gut microbiota and lifestyle factors such as diet and physical activity (PA) [3] [4] [5].

From a dietary perspective, dietary fiber and resistant starch (RS) are associated with decreased risk of developing several chronic diseases and have frequently been shown to significantly change the bacteria present within an individual's gastrointestinal system [6] [7] [8]. The assessment of breath hydrogen concentration has been used as an indirect evaluation of gut bacteria metabolism of dietary fiber [9] [10]. Specifically, one study that employed a cross-over design among healthy subjects, reported that consuming RS increased gut fermentation as evidenced by increased breath hydrogen concentration in as little as seven days [11]. Additionally, a double-blinded cross-over study in healthy human subjects demonstrated that RS type 4 elicited significant microbiome changes in as little as two to three weeks [12]. Results from a single-blinded cross-over study revealed that high RS intake (60 grams in $24 \mathrm{hrs)} \mathrm{significantly}$ increased breath hydrogen among healthy adult individuals [13]. However, there are limited data available to indicate whether breath hydrogen increases more acutely, such as in within the postprandial period, immediately following RS consumption.

Unlike RS, there is little evidence including the effects of PA on gut bacteria within humans. However, a cross-sectional study testing rugby players found that PA altered the composition of gut microbiota compared to a control group at one-time point [3]. This study analyzed fecal microbiota composition and showed 22 distinct phyla with a higher diversity of gut microbiota in the rugby players as compared to the control group. Having a higher diversity of gut microbiota is commonly found among lean individuals compared to obese individuals, potentially indicating better health outcomes [14] [15]. However, due to the cross-sectional nature of the study, causality for PA on gut microbiota could not be determined.

There are few studies that have been designed to better understand the impact of PA at different levels of RS consumption on gut microbiota. A better understanding of the effects of PA on gut fermentation following consumption of different levels of RS is important given the recommendations to include both in a healthy lifestyle [16] [17]. Therefore, the purpose of the current study was to investigate the possible effects of chronic PA on breath hydrogen production and blood glucose, at different dosages of RS, indicating potential metabolic health effects. We hypothesized that more physically active individuals would exhibit greater breath hydrogen production due to a potentially greater diversity of gut microbiota, and decreased two-hour blood glucose response as compared to the less physically active group. We utilized a simple, fast and non-invasive measure of gut fermentation (breath hydrogen production), and also assessed the acute 
postprandial period, to further explore the interaction between gut fermentation and metabolic health in a true-to-life scenario.

\section{Methods}

Twenty healthy young participants ( $9 \mathrm{M} / 11 \mathrm{~F}$; age: $24.5 \pm 3.4 \mathrm{yrs})$ were recruited from the local area. All participants were non-smokers, free from ongoing dysmetabolic conditions, and had not used antibiotics for at least the past three months. Participants were recruited from posted flyers and contacted a research assistant via phone or email. Participants were screened for whether they met the American College of Sport Medicine (ACSM) physical activity guidelines of 30 minutes or more of moderate-to-vigorous physical activity (MVPA) five times a week, via a short form International physical activity questionnaire (IPAQ-SF) for seven days [17] [18]. We further assessed PA using Actical accelerometers (Respironics Inc., Bend, OR, USA) as an objective PA assessment. Participants wore accelerometers on their wrist, and based on seven consecutive days of accelerometer data, were divided into two different groups by median split: a "more active group" (MA, more than 160 minutes per day MVPA) or "less active group" (LA, less than 116 minutes per day MVPA). The study was approved by the Institutional Review Board of Human Subjects at Kansas State University, Manhattan, Kansas (protocol \#7901).

All participants were required to attend three appointments at the Physical Activity and Nutrition Clinical Research Consortium facility (PAN-CRC). On the first visit, participants provided both oral and written informed consent for the study. Then, participants listened to a brief introduction of the study, provided required information through documents (medical history form, IPAQ, 3-day food record), and accelerometers were attached on their non-dominant wrist. Each participant's height and weight, used to calculate Body Mass Index (BMI), were measured. Three-day food record data were analysed using Nutritionist Pro software by the same technician (Axxya Systems LLC, WA, USA). For the food records, participants were instructed to write down the food items consumed, the brand/type of food, and portion sizes based on the instructions.

Participants each completed two separate meal sessions, following a $10-12$ hour fast and exercise avoidance for 48 hours. At each meal session, participants consumed a commercially available breakfast bowl (Great Value, Walmart, AR, USA), with the main ingredients being potatoes, eggs, cheese, and sausage. The amount of food consumed was calculated based on participant's body weight (10 $\mathrm{kcal} / \mathrm{kg}$ ). This dose was chosen based on the previous literature [19] to represent a high-fat meal ( $58 \%$ fat), with kcals ranging from 466 to $1002 \mathrm{kcals}$. Either $5 \mathrm{~g}$ or $25 \mathrm{~g}$ of RS type 4 powder (MGP Ingredients, KS, USA) was added to a lemonade beverage that was consumed along with the breakfast bowl (Minute Maid Premium Lemonade, $250 \mathrm{~mL}$, The Coca-Cola Company, GA, USA). Participants were blinded to the RS dosage, and the meal was consumed within 20 minutes 
on each visit.

For each meal session, breath hydrogen $\left(\mathrm{H}_{2}\right)$ was measured at baseline and every hour up to the fourth hour using Quintron Breath Tracker Analyzer (Breath Tracker SC, Quintron Instrument Company, Inc., WI, USA). After the fourth hour, measurements were taken every 30 minutes. Exhaled breath collection was performed according to manufacturer standards (Alveosampler, cat.\#: QT00827-P, Quintron Instrument Company, Inc., WI, USA) and processed within 2 hours of collection. The present study assessed the fourth-hour breath hydrogen AUC as well, due to the consideration of individual variability in oro-cecal transit time (OCTT) between 192 and 232 minutes following consumption of a solid meal [20]. Additionally, a previous study measured the breath hydrogen AUC from 180 minutes until the end of the study [5]. To assess glucose, there were seven serial finger pricks at baseline, 15, 30, 45, 60, 90, and 120 minutes after the consumption of the test meal, performed according to standard protocol using a Bayer Countour blood glucose meter (Bayer HealthCare LLC, Mishawaka IN, USA) [21].

Data are presented as Mean \pm SD in tables and Mean \pm SE in figures. The sample size was determined based on the previous literature, indicating that 10 to 20 subjects were required for adequate power to detect differences in breath hydrogen response or blood glucose, respectively [5] [13]. Shapiro-Wilk tests were performed for determining the normality distribution of each variable [22]. A Mann-Whitney $U$ test and student $t$-test were performed for determining differences between the groups. Spearman correlations were conducted for MVPA and self-report MVPA as well as with hydrogen data with PA level. The incremental area under the curve (iAUC) for postprandial glucose $(\mathrm{mmol} / \mathrm{l} \times 2 \mathrm{hr})$, and total area under the curve (tAUC) for breath hydrogen ( $\mathrm{ppm} \times 6 \mathrm{hr}$ ), fourth hour AUC breath hydrogen were calculated using the trapezoid model with v6.0 Prism (GraphPad Software, Inc., La Joya, CA, USA). Two-way mixed analysis of variance (ANOVA) was performed for postprandial glucose iAUC and log-transformed breath hydrogen AUC and fourth-hour hydrogen AUC, with RS and PA as two independent factors. Three-way ANOVA with repeated measures was performed for the postprandial glucose response. Freidman's tests and Wilcoxon signed rank tests were conducted for breath hydrogen data analysis within the groups and between the groups at the different level of RS. All the above statistics were completed using IBM SPSS Statistics 23 (SPSS Inc., Chicago, IL, USA). The level of significance was set at $p<0.05$.

\section{Results}

Participant characteristics and PA status are presented in Table 1. A total of eleven females and nine males completed the study. The more active (MA) group was older than the less active (LA) group $(p<0.05)$. The other descriptive variables were not significantly different between groups. The MA group achieved more daily steps and MVPA (19,728 steps/day and 204 mins/day) compared 
with the LA group (11,486 steps/day and 96 mins/day) $(p<0.05)$. Data from the 3 -day food records are displayed by the group in Table 2 . There were no differences in dietary intake between the two groups.

Postprandial breath hydrogen responses are displayed in Figure 1(a). Postprandial breath hydrogen total AUC (tAUC) data are displayed in Table 3. Based on the two-way ANOVA results, there was no interaction effect for postprandial

Table 1. Participant characteristics and physical activity (PA) summary.

\begin{tabular}{|c|c|c|c|c|c|}
\hline \multirow[b]{2}{*}{ Sex } & \multicolumn{2}{|c|}{ More Active group $(n=10)$} & \multicolumn{2}{|c|}{ Less Active group $(\mathrm{n}=10)$} & \multirow{2}{*}{$\frac{p \text {-value }}{0.178}$} \\
\hline & Male $(\mathrm{n}=3)$ & Female $(\mathrm{n}=7)$ & Male $(n=6)$ & Female $(n=4)$ & \\
\hline & \multicolumn{2}{|c|}{ Mean \pm SD } & \multicolumn{2}{|c|}{ Mean \pm SD } & \\
\hline Age (yrs) & \multicolumn{2}{|c|}{$26.0 \pm 3.5$} & \multicolumn{2}{|c|}{$22.8 \pm 2.4^{*}$} & 0.300 \\
\hline BMI $\left(\mathrm{kg} / \mathrm{m}^{2}\right)$ & \multicolumn{2}{|c|}{$23.75 \pm 3.84$} & \multicolumn{2}{|c|}{$25.33 \pm 4.12$} & 0.384 \\
\hline Height (m) & \multicolumn{2}{|c|}{$1.70 \pm 0.09$} & \multicolumn{2}{|c|}{$1.67 \pm 0.08$} & 0.565 \\
\hline Weight (kg) & \multicolumn{2}{|c|}{$69.0 \pm 16.5$} & \multicolumn{2}{|c|}{$71.8 \pm 17.1$} & 0.717 \\
\hline $\begin{array}{l}\text { Baseline Glucose } \\
(\mathrm{mmol} / \mathrm{L})\end{array}$ & \multicolumn{2}{|c|}{$4.76 \pm 0.38$} & \multicolumn{2}{|c|}{$4.82 \pm 0.41$} & 0.677 \\
\hline $\begin{array}{c}\text { Baseline Hydrogen } \\
(\mathrm{ppm})\end{array}$ & \multicolumn{2}{|c|}{$10 \pm 13$} & \multicolumn{2}{|c|}{$11 \pm 11$} & 0.436 \\
\hline Steps (day) & \multicolumn{2}{|c|}{$19728 \pm 5256$} & \multicolumn{2}{|c|}{$11486 \pm 3997^{\star}$} & 0.001 \\
\hline MVPA (mins/day) & \multicolumn{2}{|c|}{$204.2 \pm 41.2$} & \multicolumn{2}{|c|}{$95.8 \pm 24.1^{*}$} & $<0.001$ \\
\hline (mins/week) & \multicolumn{2}{|c|}{$1429.4 \pm 288.5$} & \multicolumn{2}{|c|}{$670.6 \pm 168.9$} & \\
\hline $\begin{array}{l}\text { MVPA (self-report) } \\
\text { (mins/week) }\end{array}$ & \multicolumn{2}{|c|}{$141.5 \pm 173.6$} & \multicolumn{2}{|c|}{$109.5 \pm 125$} & 0.853 \\
\hline
\end{tabular}

${ }^{*}$ Less active group is significantly different from more active group $(p<0.05)$ MVPA: moderate-to-vigorous physical activity.

Table 2. Three-day food record dietary summary.

\begin{tabular}{cccc}
\hline & $\begin{array}{c}\text { More Active Group } \\
(\mathrm{N}=10)\end{array}$ & $\begin{array}{c}\text { Less Active Group } \\
(\mathrm{N}=10)\end{array}$ & $p$-value \\
\cline { 2 - 4 } & Mean $\pm \mathrm{SD}$ & Mean $\pm \mathrm{SD}$ & \\
\hline Total KJ & $8002 \pm 2680$ & $8182 \pm 3483$ & 0.718 \\
- Kilocalorie & $1912 \pm 641$ & $2034 \pm 832$ & \\
Carbohydrate (g) & $202 \pm 70$ & $252 \pm 77$ & 0.146 \\
g/kg bw & $3.1 \pm 1.4$ & $3.5 \pm 0.8$ & 0.105 \\
\% energy & $44.0 \pm 11.9$ & $51.9 \pm 7.8$ & 0.095 \\
Protein (g) & $89 \pm 36$ & $81 \pm 44$ & 0.481 \\
g/kg bw & $1.34 \pm 0.53$ & $1.10 \pm 0.50$ & 0.329 \\
\% energy & $18.96 \pm 4.30$ & $15.29 \pm 4.22$ & 0.071 \\
Fat (g) & $86 \pm 53$ & $78 \pm 46$ & 0.739 \\
g/kg bw & $1.26 \pm 0.71$ & $1.05 \pm 0.48$ & 0.447 \\
\% energy & $38.06 \pm 10.28$ & $32.30 \pm 6.05$ & 0.144 \\
Fiber (g) & $17 \pm 10$ & $14 \pm 10$ & 0.481 \\
g/1000 kcal & $8.80 \pm 4.15$ & $6.92 \pm 2.97$ & 0.436 \\
\hline
\end{tabular}

bw: body weight. 
breath hydrogen AUC between PA groups at different levels of RS dosage, and no interaction effect in fourth-hour breath hydrogen AUC between PA groups at the different levels of RS dosage ( $p=0.281$ ). Additionally, in the LA group, $5 \mathrm{~g}$ of RS showed higher fourth hour AUC compared to $25 \mathrm{~g}$, however this result was not significant according to the a priori p-value cutpoint $\left(\mathrm{X}^{2}(1)=3.600, p=\right.$ 0.058). There was an inverse correlation for AUC hydrogen at the $25 \mathrm{~g}$ RS dose and MVPA $(r=-0.869, \mathrm{~N}=10, p=0.001)$.

Postprandial glucose $(\mathrm{mmol} / \mathrm{L})$ responses following the two meal sessions are displayed in Figure 1(b). Based on the three-way RM ANOVA, there was no significant interaction effect between RS dose and PA group. However, there was a significant Time $\times$ RS dose interaction effect in the glucose response $(p<0.05)$, indicating the higher dose of RS showed less fluctuation throughout the period.

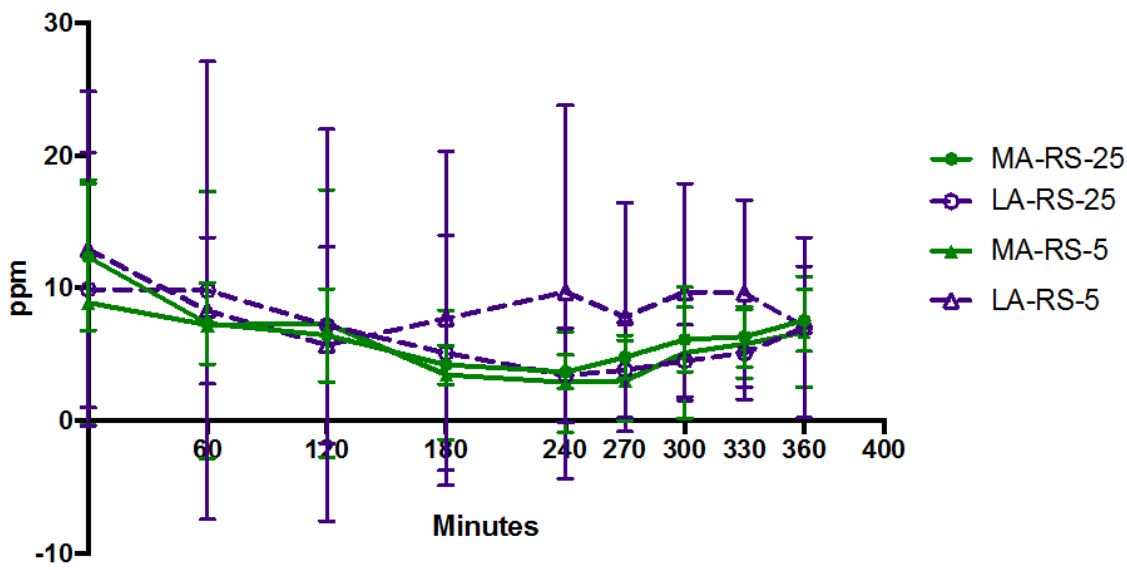

(a)

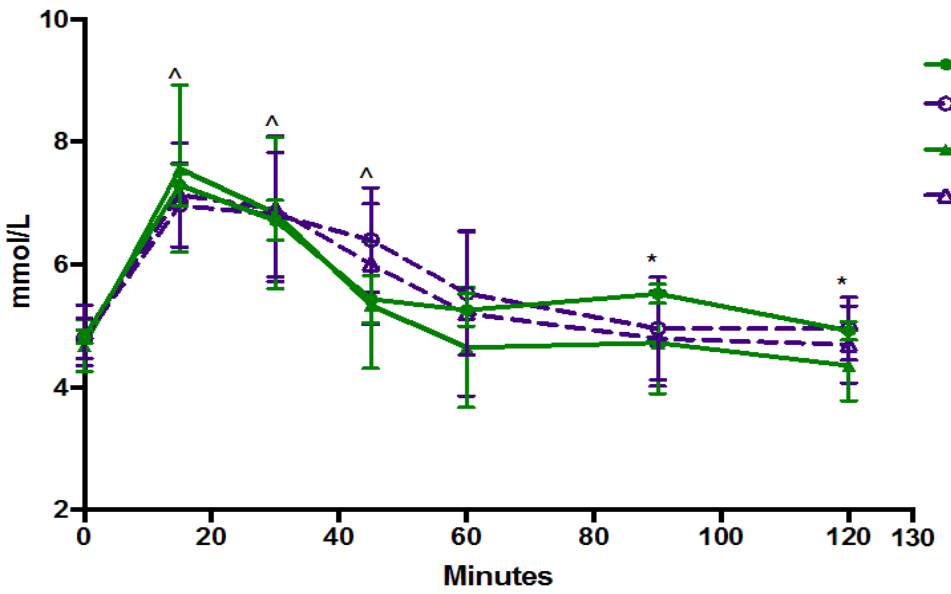

MA-RS-25

$\rightarrow$ LA-RS-25

- MA-RS-5

$\sim$ LA-RS-5

(b)

Figure 1. Postprandial breath hydrogen and glucose responses. (a) Postprandial breath hydrogen for 360 minutes, (b) Postprandial glucose for 120 minutes for More active group with $25 \mathrm{~g}$ of resistant starch received (MA-RS-25) and $5 \mathrm{~g}$ of resistant starch received (MA-RS-5), and Less active group with $25 \mathrm{~g}$ of resistant starch received (LA-RS-25) and 5 $\mathrm{g}$ of resistant starch received (LA-RS-5), Mean $\pm \mathrm{SE}$, ${ }^{\star} \mathrm{MA}$ with $25 \mathrm{~g}$ intake significantly different from MA with $5 \mathrm{~g}$ intake $(p<0.05) \wedge$ significant time effect compared to baseline $(p<0.05)$. 
Table 3. Incremental area under the curve (iAUC) for postprandial glucose and postprandial breath hydrogen area under the curve (tAUC) by group and RS dosage.

\begin{tabular}{|c|c|c|c|c|c|c|c|}
\hline & \multicolumn{2}{|c|}{$\begin{array}{l}\text { More Active group } \\
\qquad(\mathrm{n}=10)\end{array}$} & \multicolumn{2}{|c|}{$\begin{array}{l}\text { Less Active group } \\
\qquad(\mathrm{n}=10)\end{array}$} & \multirow[t]{2}{*}{$\mathrm{RS} \times \mathrm{PA}^{\&}$} & \multirow[t]{2}{*}{$\mathrm{RS}^{\&}$} & \multirow[t]{2}{*}{$\mathrm{PA}^{\&}$} \\
\hline & $25 \mathrm{~g}$ RS & $5 \mathrm{~g}$ RS & $25 \mathrm{~g}$ RS & $5 \mathrm{~g}$ RS & & & \\
\hline iAUC glucose ${ }^{1}$ & $118.2 \pm 52.7$ & $125.6 \pm 40.7$ & $126.6 \pm 45.3$ & $122.6 \pm 62.7$ & 0.659 & 0.894 & 0.891 \\
\hline tAUC Hydrogen ${ }^{2}$ & $2424 \pm 2100$ & $2129 \pm 2065$ & $2283 \pm 3110$ & $3045 \pm 2962$ & & & \\
\hline AUC Hydrogen ${ }^{3}$ & $3.2 \pm 0.5$ & $3.2 \pm 0.4$ & $3.2 \pm 0.4$ & $3.3 \pm 0.4$ & 0.369 & 0.415 & 0.622 \\
\hline $\begin{array}{c}\text { AUC Hydrogen } \\
\left(\%^{5}\right)\end{array}$ & $\begin{array}{c}963 \pm 1096 \\
(40.1)\end{array}$ & $\begin{array}{l}524 \pm 405 \\
\quad(40.3)\end{array}$ & $\begin{array}{l}659 \pm 462 \\
\quad(30.6)\end{array}$ & $\begin{array}{c}1053 \pm 879 \\
\quad(39.0)\end{array}$ & & & \\
\hline
\end{tabular}

Mean $\pm \mathrm{SD}{ }^{1}$ units for iAUC: $\mathrm{mmol} /$ minutes $/ \mathrm{L} ;{ }^{2}$ units for total AUC: $\mathrm{ppm} /$ minutes; ${ }^{3} \log 10$ transformed data were used; ${ }^{4}$ fourth hour AUC hydrogen calculation: 240 minutes (baselines) - 360 minutes; ${ }^{5}$ denotes the proportion of fourth hour AUC in total AUC hydrogen in percentage; ${ }^{8}$ indicates two way mixed analysis of variance was conducted: interaction effect of RS and PA, main effect from RS and PA, $p$-values all $>0.05$. All of the AUC hydrogen variables were calculated with $\mathrm{ppm}$ * minutes.

Among LA, there was no difference in glucose response at 90 and 120 minutes. Postprandial glucose iAUC is represented in Table 3. Additionally, there was a significant time effect for glucose values at 15, 30 and 45 minutes compared to the baseline $(p<0.05)$. At 90 and 120 minutes following meal consumption, MA showed a higher glucose response for the $25 \mathrm{~g}$ dosage (5.53 \pm 0.50 vs. $4.73 \pm 0.83$ and $4.92 \pm 0.46$ vs. $4.36 \pm 0.58)$ compared to the $5 \mathrm{~g}$ dosage $(p<0.05)$. However, LA did not show any difference between the $5 \mathrm{~g}$ or $25 \mathrm{~g}$ RS dosage.

\section{Discussion}

We hypothesized that chronic level of PA would have an impact on the breath hydrogen and blood glucose responses to different doses of RS. Our results support the null hypothesis, indicating that there was no effect for chronic level of PA on breath hydrogen or glucose responses to high or low doses of RS during the acute postprandial period in apparently healthy adults. To our knowledge, there are no previous studies which have examined the impact of chronic PA level on breath hydrogen production, either in the fasted or postprandial state. With regard to the glucose response, there was a significant difference at 90 and 120 minutes when comparing the $25 \mathrm{~g}$ RS dosage to the $5 \mathrm{~g}$ RS dosage in the MA group, supporting a potentially beneficial effect of the high level of RS among the more active participants.

Numerous studies have shown that a high dose of RS elicits a large breath hydrogen response 24 hours or later [11] [13]. The current study investigated breath hydrogen for 6 hours post-meal, potentially explaining the null findings, since evidence suggests that additional time is required to detect breath hydrogen [20] [23]. It is possible that our assessment was insufficient in duration for accurately observing the production of hydrogen from gut to oral assessment. Thus, there is a discrepancy between our results and results from a previous study that examined the acute post-exercise breath hydrogen response. Participants exercised for 5 minutes on a treadmill at $10 \mathrm{~km} / \mathrm{h}$ with an incline of $20 \%$, 
and results indicated a consistently increased breath hydrogen response when comparing the breath hydrogen tAUC and third hour AUC until the end of the study [5]. Despite the lack of differences in our study, the proportion of hydrogen produced between hours 4 and 6 reached almost $40 \%$ of the total hydrogen production in MA and LA, indicating a greater time requirement for accurately assessing gut fermentation. This result suggests that breath hydrogen response is likely initiated at least 4 hours after a high RS intake.

The current study results indicated no difference in glucose iAUC between the MA and LA participants; however, there was a higher glucose response in the MA for the $25 \mathrm{~g}$ RS dosage as compared with the $5 \mathrm{~g}$ RS dosage at 90 and 120 minutes. This result may be due to a protective effect of more RS type 4, in the gut, indicating a prolonged time for digestion and inflation of stomach, and thus possibly providing longer satiety. Additionally, the MA group had a higher peak glucose at 15 minutes for the $5 \mathrm{~g}$ RS dosage compared to $25 \mathrm{~g}$ RS dosage, pointing to a potentially beneficial effect of RS [21] [24]. However, in the LA, we saw the opposite trend with high glucose levels at peak. Overall, RS type 4 seemed to be advantageous for maintaining glucose homeostasis within the MA group, but not in the LA group.

We believe our study is novel and contributes to the larger body of research knowledge regarding the interactions between PA and diet. Particularly, examining the effect of chronic PA status on acute breath hydrogen production and blood glucose following a high and low dosage of RS was innovative. Our study utilized a true-to-life meal which was relative to the size of the individual. Also, use of the objective PA assessment substantially strengthened the group assignment by PA status. However, there are limitations to be considered when interpreting the results. Despite the utilization of participants' self-reported levels of PA for screening for the purpose of recruiting sufficiently and insufficiently active participants, according to objective accelerometry, both groups exceeded the ACSM physical activity guideline recommendation of 150 minutes per week of MVPA. We observed that four out of ten of the LA group subjects preferred to commute by walking and biking, with the rest commuting by automobiles. It is possible that college students who frequently engage in walking between classes and home, may not perceive these activities as MVPA according to IPAQ definitions. Inferring from a very low correlation between objective MVPA and self-report MVPA, it is possible that wrist-based accelerometers may have overestimated MVPA in this population, and wrist-based accelerometer cut-points may need to be revised. However, there was a consistency with regard to some additional unexpected findings for the LA group. Especially, within the LA group at the $5 \mathrm{~g}$ RS dosage. An inverse correlation was found between breath hydrogen AUC and MVPA which is the opposite of what we expected. These results may be due to a small sample size combined with a high variability in the LA group's postprandial breath hydrogen, reflecting the complexity of analyzing breath hydrogen in human participants. 


\section{Conclusion}

In conclusion, chronic PA levels did not modify the acute breath hydrogen or blood glucose responses to high or low RS doses during a six-hour assessment period. However, given that the duration of the assessment was likely insufficient to adequately understand breath hydrogen responses following the consumption of different RS doses, further studies are warranted. In addition, future studies may need to include more participants in order to generalize the effects of chronic PA with different dosage of RS on metabolic health outcomes.

\section{Conflicts of Interest}

The authors declare no conflicts of interest regarding the publication of this paper.

\section{References}

[1] Sanz, Y., Santacruz, A. and Gauffin, P. (2010) Gut Microbiota in Obesity and Metabolic Disorders. Proceedings of the Nutrition Society, 69, 434-441. https://doi.org/10.1017/S0029665110001813

[2] Tremaroli, V. and Backhed, F. (2012) Functional Interactions between the Gut Microbiota and Host Metabolism. Nature, 489, 242-249. https://doi.org/10.1038/nature11552

[3] Clarke, S.F., Murphy, E.F., O’Sullivan, O., Lucey, A.J., Humphreys, M., Hogan, A., Hayes, P., O’Reilly, M., Jeffery, I.B., Wood-Martin, R., Kerins, D.M., Quigley, E., Ross, R.P., O’Toole, P.W., Molloy, M.G., Falvey, E., Shanahan, F. and Cotter, P.D. (2014) Exercise and Associated Dietary Extremes Impact on Gut Microbial Diversity. Gut, 63, 1913-1920. https://doi.org/10.1136/gutjnl-2013-306541

[4] Turnbaugh, P.J., Backhed, F., Fulton, L. and Gordon, J.I. (2008) Diet-Induced Obesity Is Linked to Marked But Reversible Alterations in the Mouse Distal Gut Microbiome. Cell Host Microbe, 3, 213-223. https://doi.org/10.1016/j.chom.2008.02.015

[5] Ehrenpreis, E.D., Swamy, R.S., Zaitman, D. and Noth, I. (2002) Short Duration Exercise Increases Breath Hydrogen Excretion after Lactulose Ingestion: Description of a New Phenomenon. American Journal of Gastroenterology, 97, 2798-2802. https://doi.org/10.1111/j.1572-0241.2002.07025.x

[6] Anderson, J.W., Baird, P., Davis, R.H., Ferreri, S., Knudtson, M., Koraym, A., Waters, V. and Williams, C.L. (2009) Health Benefits of Dietary Fiber. Nutrition Reviews, 67, 188-205. https://doi.org/10.1111/j.1753-4887.2009.00189.x

[7] Nichenametla, S.N., Weidauer, L.A., Wey, H.E., Beare, T.M., Specker, B.L. and Dey, M. (2014) Resistant Starch Type 4-Enriched Diet Lowered Blood Cholesterols and Improved Body Composition in a Double Blind Controlled Cross-Over Intervention. Molecular Nutrition \& Food Research, 58, 1365-1369.

https://doi.org/10.1002/mnfr.201300829

[8] Papathanasopoulos, A. and Camilleri, M. (2010) Dietary Fiber Supplements: Effects in Obesity and Metabolic Syndrome and Relationship to Gastrointestinal Functions. Gastroenterology, 138, 65-72e1-2. https://doi.org/10.1053/j.gastro.2009.11.045

[9] Bond Jr., J.H. and Levitt, M.D. (1972) Use of Pulmonary Hydrogen (H2) Measurements to Quantitate Carbohydrate Absorption: Study of Partially Gastrectomized Patients. Journal of Clinical Investigation, 51, 1219-1225.

https://doi.org/10.1172/JCI106916 
[10] Rumessen, J.J. (1992) Hydrogen and Methane Breath for Evaluation of Resistant Carbohydrates. European Journal of Clinical Nutrition, 46, S77-S90.

[11] van Munster, I.P., de Boer, H.M., Jansen, M.C., de Haan, A.F., Katan, M.B., van Amelsvoort, J.M. and Nagengast, F.M. (1994) Effect of Resistant Starch on Breath-Hydrogen and Methane Excretion in Healthy Volunteers. The American Journal of Clinical Nutrition, 59, 626-630. https://doi.org/10.1093/ajcn/59.3.626

[12] Martinez, I., Kim, J., Duffy, P.R., Schlegel, V.L. and Walter, J. (2010) Resistant Starches Types 2 and 4 Have Differential Effects on the Composition of the Fecal Microbiota in Human Subjects. PLoS ONE, 5, e15046. https://doi.org/10.1371/journal.pone.0015046

[13] Robertson, M.D., Currie, J.M., Morgan, L.M., Jewell, D.P. and Frayn, K.N. (2003) Prior Short-Term Consumption of Resistant Starch Enhances Postprandial Insulin Sensitivity in Healthy Subjects. Diabetologia, 46, 659-665.

https://doi.org/10.1007/s00125-003-1081-0

[14] Turnbaugh, P.J., Hamady, M., Yatsunenko, T., Cantarel, B.L., Duncan, A., Ley, R.E., Sogin, M.L., Jones, W.J., Roe, B.A., Affourtit, J.P., Egholm, M., Henrissat, B., Heath, C., Knight, R. and Gordon, J.I. (2009) A Core Gut Microbiome in Obese and Lean Twins. Nature, 457, 480-484. https://doi.org/10.1038/nature07540

[15] Denou, E., Marcinko, K., Surette, M.G., Steinberg, G.R. and Schertzer, J.D. (2016) High-Intensity Exercise Training Increases the Diversity and Metabolic Capacity of the Mouse Distal Gut Microbiota during Diet-Induced Obesity. American Journal of Physiology-Endocrinology and Metabolism, 310, E982-E993. https://doi.org/10.1152/ajpendo.00537.2015

[16] Murphy, M.M., Douglass, J.S. and Birkett, A. (2008) Resistant Starch Intakes in the United States. Journal of the American Dietetic Association, 108, 67-78. https://doi.org/10.1016/j.jada.2007.10.012

[17] Thompson, P.D., Arena, R., Riebe, D., Pescatello, L.S. and American College of Sports Medicine (2013) ACSM's New Pre-Participation Health Screening Recommendations from ACSM's Guidelines for Exercise Testing and Prescription, Ninth Edition. Current Sports Medicine Reports, 12, 215-217. https://doi.org/10.1249/JSR.0b013e31829a68cf

[18] Craig, C.L., Marshall, A.L., Sjöström, M., Bauman, A.E., Booth, M.L., Ainsworth, B.E., Pratt, M., Ekelund, U., Yngve, A., Sallis, J.F. and Oja, P. (2003) International Physical Activity Questionnaire: 12-Country Reliability and Validity. Medicine \& Science in Sports \& Exercise, 35, 1381-1395. https://doi.org/10.1249/01.MSS.0000078924.61453.FB

[19] Kurti, S.P., Rosenkranz, S.K., Levitt, M., Cull, B.J., Teeman, C.S., Emerson, S.R. and Harms, C.A. (2015) Does Moderate Intensity Exercise Attenuate the Postprandial Lipemic and Airway Inflammatory Response to a High-Fat Meal? BioMed Research International, 2015, Article ID: 647952. https://doi.org/10.1155/2015/647952

[20] Read, N.W., Fisher, D., Holgate, A.M., Kime, N.D., Mitchell, M.A., Reeve, A.M., Roche, T.B. and Walker, M. (1980) Transit of a Meal through the Stomach, Small Intestine, and Colon in Normal Subjects and Its Role in the Pathogenesis of Diarrhea. Gastroenterology, 79, 1276-1282. https://doi.org/10.1016/0016-5085(80)90925-7

[21] Lin, C.H., Chang, D.M., Wu, D.J., Peng, H.Y. and Chuang, L.M. (2015) Assessment of Blood Glucose Regulation and Safety of Resistant Starch Formula-Based Diet in Healthy Normal and Subjects with Type 2 Diabetes. Medicine (Baltimore), 94, e1332. https://doi.org/10.1097/MD.0000000000001332

[22] Ghasemi, A. and Zahediasl, S. (2012) Normality Tests for Statistical Analysis: A 
Guide for Non-Statisticians. International Journal of Endocrinology and Metabolism, 10, 486-489. https://doi.org/10.5812/ijem.3505

[23] Gasbarrini, A., Corazza, G.R., Gasbarrini, G., Montalto, M., Di Stefano, M., Basilisco, G., Parodi, A., Usai-Satta, P., Vernia, P., Anania, C., Astegiano, M., Barbara, G., Benini, L., Bonazzi, P., Capurso, G., Certo, M., Colecchia, A., Cuoco, L., Di Sario, A., Festi, D., Lauritano, C., Miceli, E., Nardone, G., Perri, F., Portincasa, P., Risicato, R., Sorge, M., Tursi, A. and 1st Rome H2-Breath Testing Consensus Conference Working Group (2009) Methodology and Indications of H2-Breath Testing in Gastrointestinal Diseases: The Rome Consensus Conference. Alimentary Pharmacology \& Therapeutics, 29, 1-49.

https://doi.org/10.1111/j.1365-2036.2009.03951.x

[24] Brewer, L.R., Weber, C., Haub, M., Cai, L. and Shi, Y.C. (2015) Glycemic Response and Fermentation of Crystalline Short Linear Alpha-Glucans from Debranched Waxy Maize Starch. Journal of Agricultural and Food Chemistry, 63, 9528-9535. https://doi.org/10.1021/acs.jafc.5b03632 